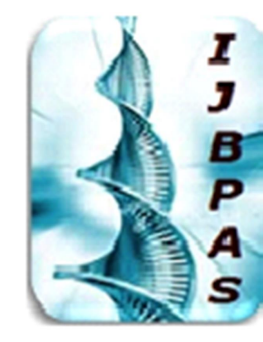

International Journal of Biology, Pharmacy and Allied Sciences (IJBPAS) 'A Bridge Betuen Caboratory and QRendo'

www.ijbpas.com

\title{
PARASTOMAL HERNIAS: MANAGEMENT STRATEGIES
}

\section{PACHANI $\mathbf{N}^{1 *}$, NANIWADEKAR RG ${ }^{2}$ AND TEWANI VH ${ }^{3}$}

1: Junior Resident, Department of Surgery, Krishna Institute of Medical Sciences Deemed To Be University, Karad, Maharashtra (India)

2: Professor, Department of Surgery, Krishna Institute of Medical Sciences Deemed To Be

$$
\text { University, Karad, Maharashtra (India) }
$$

3: 2) Assisst. Professor, Department of Surgery, Krishna Institute of Medical Sciences

Deemed To Be University, Karad, Maharashtra (India)

*Corresponding Author: Dr. Nevil Nitin Pachani: E Mail: nevilpachani@rocketmail.com

Received $12^{\text {th }}$ July 2021; Revised $14^{\text {th }}$ Aug. 2021; Accepted $27^{\text {th }}$ Oct. 2021; Available online $15^{\text {th }}$ Feb. 2022

https://doi.org/10.31032/IJBPAS/2022/11.2.1019

\section{INTRODUCTION}

Stomas have been created by surgeons as a diversion of feces since the early 1800s. Morbidity and mortality related to stoma creation has progressively decreased with the advancement of modern therapy. Morbidity related to stoma was estimated between $2.9 \%$ to $81.1 \%$ in India [1]. Stomal issues, include skin excoriation, leakage from devices, dehydration from high output stomas, bowel obstruction, prolapse, and parastomal hernia are common impediments to this life saving procedure. Parastomal hernias are of an interest as their further management needs surgical intervention.

\begin{tabular}{|c|c|}
\multicolumn{2}{|c|}{ Types of Parastomal hernias } \\
\hline Subtype & Through muscular plane \\
\hline 1) Interstitial & Through subcutaneous tissue \\
\hline 2) Subcutaneous & Through layers of prolapsed bowel \\
\hline 3) Intrastomal & Through emerging intestinal wall and everted intestinal layer \\
\hline 4) Peristomal & Thersed \\
\hline
\end{tabular}

\section{Definition and clinical features}

A parastomal hernia is a type of incisional hernia that allows protrusion of abdominal contents through the abdominal wall defect created during ostomy formation [2].

Patients generally present with a bulge at or around the stoma site. This may or may not 
be associated with pain. Pain may have multiple causes including dragging type due to hernia, cramping of bowel or irritation of skin by stomal secretions due to improper fitting of the appliance. Misfit of appliance is another complaint. Patients may rarely present with strangulation of bowel with symptoms of obstruction.

\section{Management}

Most patients with a parastomal hernia do not have symptoms that are sufficient enough to warrant repair. For patients with mild symptoms, conservative management is ideal. This conservative management includes changing of appliance type, shape. Stomal nursing care is of prime importance for improved quality of care [3].

Conservative management is not always viable. Though recurrence rates are high, ranging from 2.9 to $69.4 \%$ depending on the type of repair [4], surgical management is the only management for moderate to severe symptomatic cases.

\section{1) Simple Fascial repair}

This technique is a modality of the past due to high recurrence rates of upto $69.4 \%$ [4].After incision sac is reduced and musculoperitoneal layer is sutured [5]. Simple fascial has even worse re-recurrence rates of $100 \%$ [6].

2) Stoma Relocation
Although theoretically a sound process, second site herniation to the original is common. However it has been proven to be superior to simple fascial repair. There is a risk of repeat laparotomy in translocation. It is recommended that the new stoma be places on the opposite side of midline to prevent recurrence [7].

\section{3) Mesh repair}

Mesh repair has been the preferred technique due to reduced recurrence rates. Synthetic meshes have less recurrence rates but causes other complications like fistula formation due to mesh erosion, adhesions, risk of infecting mesh subsequently causing infection of bowel [8].

PTFE and biological mesh have advantage of not causing above problems. PTFE may not adhere to bowel but has a problem of shrinkage [9]. Biological meshes are expensive, have low chances of infection but may cause seromas [10].

Described recurrence rates for mesh repair are less than $20 \%$ for both synthetic and biological meshes [4]. Placement of meshes include inlay, onlay, retromuscular and intraperitoneal 
Onlay method involves mesh placement over anterior rectus sheath and external oblique aponeurosis.

Inlay is suturing mesh to fascial defects and has a high failure rate.

Intraperitoneal approach is one where mesh is placed over peritoneum.

Retromuscular approach fixes mesh between rectus muscle and posterior rectus sheath.

Sugarbaker and keyhole techniques were used for laproscopic approach of parastomal hernia repairs. Recently a combination of both called the Sandwich technique as recurrence of as low as 2.1\% [11].

Open and laparoscopic mesh repair had no significant difference in recurrence rates of parastomal hernias. Within the dataset of laparoscopic procedures, the Sugarbaker technique had a remarkably lower recurrence rate than the keyhole method. There was no difference in occurrence of mesh infection or other postoperative morbidity between any of the mesh repair subsets. Studies performed after this meta-analysis have had similar findings [12].

4) Prophylactic Mesh
A mesh placed prophylactically has been proposed to help patients with high risk prevent parastomal hernias.

Small studies have been however larger studies are required for further confirmation.

\section{CONCLUSION}

The above discussion has opened a need for a definitive management of parastomal hernias as they are a common cause of morbidity in stoma patients. An elaborate preoperative and postoperative protocol needs to be prepared on its prevention based on evidence.

The considerable morbidity of patients living stomas need a proper approach for their management as other issues including skin irritation, device failure, stenosis, obstruction also continue to haunt us.

Mesh repair has been inadvertently proved to be most effective and can be considered gold standard. More study needs to be done for prophylactic repair to prove its effectiveness. Currently early stoma reversal is the only prophylactic prevention.

\section{Acknowledgement}

Special appreciation to Ms.Rupali Salunkhe for secretarial help.

\section{REFERENCES}

[1] Malik TA, Lee MJ, Harikrishnan $\mathrm{AB}$, The incidence of stoma related morbidity-a systematic review of 
randomised controlled trials, The Annals of The Royal College of Surgeons of England, 100(7), 2018, 501-8, de Ruiter P, Bijnen AB.

[2] De Ruiter P, Bijnen AB, Successful local repair of paracolostomy hernia with a newly developed prosthetic device, International journal of colorectal disease, 7(3), 1992, 1324.

[3] Kane M, McErlean D, McGrogan M, Thompson MJ, Haughey S, Clinical protocols for stoma care: 6 , Management of parastomal hernia, Nursing standard (Royal College of Nursing (Great Britain): 1987), 18(19), 2004, 43-4.

[4] Hansson BM, Slater NJ, van der Velden AS, Groenewoud HM, Buyne OR, de Hingh IH, et al, Surgical techniques for parastomal hernia repair: a systematic review of the literature, Annals of surgery, 255(4), 2012, 685-95.

[5] Lochan R, Slater BJ, Goulbourne IA, Letter 1: Parastomal hernia (Br J Surg 2003; 90: 784-793), British Journal of Surgery, 90(10), 2003, 1306.

[6] Rubin MS, Schoetz DJ, Matthews JB, Parastomal hernia: is stoma relocation superior to fascial repair?
Archives of surgery, 129(4), 1994, 413-9.

[7] Allen-Mersh TG, Thomson JP, Surgical treatment of colostomy complications, Journal of British Surgery, 75(5), 1988, 416-8.

[8] Morris-Stiff G, Hughes LE, The continuing challenge of parastomal hernia: failure of a novel polypropylene mesh repair, Annals of the Royal College of Surgeons of England, 80(3), 1998, 184.

[9] Hansson BM, Bleichrodt RP, De Hingh IH, Laparoscopic parastomal hernia repair using a keyhole technique results in a high recurrence rate, Surgical endoscopy, 23(7), 2009, 1456-9.

[10] Peppas G, Gkegkes ID, Makris MC, Falagas ME, Biological mesh in hernia repair, abdominal wall defects, and reconstruction and treatment of pelvic organ prolapse: a review of the clinical evidence, The American Surgeon, 76(11), 2010, 1290-9.

[11] Berger $\mathrm{D}, \quad$ Bientzle $\mathrm{M}$, Polyvinylidene fluoride: a suitable mesh material for laparoscopic incisional and parastomal hernia repair! Hernia, 13(2), 2009, $167-$ 72. 
[12] Hansson BM, Morales-Conde S, Mussack T, Valdes J, Muysoms

FE, Bleichrodt RP, The laparoscopic modified Sugarbaker technique is safe and has a low recurrence rate: a multicenter cohort study, Surgical Endoscopy, 27(2), 2013, 494-500. 